\title{
CONTROLE PHOTOPÉRIODIQUE DE LA SAISON DE REPRODUCTION EN SALMONICULTURE : UNE EXPÉRIENCE PILOTE EN BRETAGNE
}

\author{
B. BRETON (1), G. MAISSE (1), E. LEMENN (2)
}

(1) I.N.R.A., Laboratoire de Physiologie des Poissons, Campus de Beaulieu, Avenue du Général Leclerc, 35042 RENNES CEDEX.

(2) Pisciculture de Meilh Livinot, 29114 BANNALEC.

\section{RÉSUMÉ}

Dans les conditions d'une pisciculture bretonne, nous avons induit, par traitement photopériodique, l'ovulation et la spermiation estivales de Truites arc-en-ciel dont la saison de reproduction normale est l'hiver.

Les résultats ont montré que :

1. Toutes les femelles et tous les mâles produisaient des gamètes l'été suivant l'hiver de 1 ère ou 2ème maturité.

2. La fertilité absolue observée lors des pontes avancées ne diffère pas de celle observée lors de la ponte hivernale normale correspondante.

3. La qualité des ovules dépend de la température d'élevage des géniteurs au moment de la période de ponte. Celle-ci doit impérativement être inférieure à $15^{\circ} \mathrm{C}$.

4. La croissance des alevins produits en été est comparable à celle des alevins produits en hiver.

5. La ponte hivernale suivant une ponte estivale avancée donne des ovules de qualité identique à celle observée lors de la précédente ponte hivernale; la fertilité absolue étant supérieure, compte tenu de la croissance des géniteurs.

6. La synchronisation des ovulations estivales par induction hormonale donne des pontes identiques en quantité et en qualité à celles des femelles non traitées.

Les conditions d'application du contrôle photopériodique de l'époque de ponte sont discutées.

L'induction hormonale de la ponte est envisagée dans le cas de température d'élevage voisine de $15^{\circ} \mathrm{C}$.

\section{SUMMARY}

Photoperiodic control of the reproductive season in fish farming : a pilot experiment in Brittany.

Under natural external conditions in Brittany ovulation and spermiation have been induced in summer after the photoperiodic treatment of a strain of rainbow-trout which normally reproduces in winter.

The following results have been obtained :

1. All the females and all the males have produced gametes the summer following their first or second reproductive season.

2. The absolute fertility of females after advanced spawning did not significantly differ from that obtained during the normal winter spawning period.

3. The eggs quality depends upon the water temperature during spawning, which must be less than $15^{\circ} \mathrm{C}$.

4. The growth of the summer fry is similar to that of the winter fry. 
5. The additional summer reproduction did not alter the eggs quality of the following winter spawning; according to the growth of the breeder, the absolute fertility was increased.

6. There was no significant difference between the quality and the quantity of eggs obtained either after hormonal synchronization of summer ovulations or after natural ovulations.

The conditions of application of the photoperiodic control of the reproduction are discussed. Above $15^{\circ} \mathrm{C}$, the hormonal induction of spawning must be considered.

\section{INTRODUCTION}

Obtenir des géniteurs matures et des pontes à quelque période que ce soit est un objectif que l'évolution des structures d'élevage et de commercialisation rend souhaitable pour les piscicultures dans les prochaines années. Ses implications en sont nombreuses et parmi elles on peut citer :

- I'utilisation maximum des installations d'élevage, y compris d'incubation et d'alevinage pendant la majeure partie de l'année.

- une production et une commercialisation continue,

- le choix de la période de reproduction la plus adaptée à des conditions d'élevage à un marché local, ou à des objectifs de production spécifiques tels que l'obtention à une date donnée d'animaux d'un poids donné aptes au passage en mer.

- la spécialisation vers des productions d'œufs, non inféodées à la saison hivernale.

- la suppression des importations d'œufs en dehors de tout contrôle de souches et contrôles vétérinaires, etc.

Il existe deux voies théoriques pour obtenir ce résultat. Une voie génétique par la sélection de souches à reproduction précoce ou tardive et une voie physiologique.

Dans ce dernier domaine, en dehors des traitements hormonaux non envisageables pour le contrôle total du cycle, les techniques proposées font appel à la modulation des facteurs du milieu et essentiellement de la température et de la photopériode.

Ces problèmes ont fait l'objet de nombreux travaux fondamentaux en laboratoire (voir revue de HTUN-HAN; 1977; SUNDARARAJ, 1982). C'est chez les Salmonidés qu'ils sont les plus nombreux. Dans cette famille, le développement de la gamétogénèse est essentiellement déterminé par une diminution de la photopériode, via la sensibilisation d'un axe endocrinien hypothalamus - hypophyse - gonade (BRETON et BILLARD, 1977; BRETON et al., 1982). . Avant que le mécanisme d'action de la photopériode sur le fonctionnement endocrinien ne soit élucidé, des manipulations photopériodiques ont permis selon les espèces et les souches d'obtenir des reproductions avancées de 2 à 3 mois et ce, dès 1937 (HOOVER et HUBBARD,1937), chez le Saumon de fontaine. Ces résultats ont depuis été étendus à la plupart des Salmonidés et transférés dans la pratique piscicole dans des pays tels que le Japon : (NOMURA, 1962) ou la GrandeBretagne (WHITEHEAD et al., 1978). En France, les travaux n'en sont restés qu'au stade laboratoire. Nous rapportons ici les résultats de deux anrées d'expérimentations à l'échelle pilote d'une pisciculture dans les conditions estivales bretonnes (faibles débits et hautes températures). Dans ce travail, nous avons recherché une ponte à contre saison totale et comparé les mérites de la synchronisation des pontes estivales par rapport aux pontes naturelles. Le devenir des reproducteurs utilisés a aussi été étudié.

\section{MATÉRIEL ET MÉTHOdE}

L'expérience s'est déroulée sur deux années consécutives de janvier 1980 à février 1982.

\section{Animaux :}

Les femelles proviennent d'un lot de Truites arc-en-ciel qui se sont reproduites pour la première fois à 2 ans, l'hiver 1979-1980. 
L'expérimentation 1980 a donc porté sur des animaux en deuxième reproduction et celle de 1981 sur des animaux de troisième reproduction. A chaque fois, les animaux ont été sélectionnés parmi ceux ayant pondu dans la semaine précédant le début du traitement photopériodique. Les mâles ont la même origine que les femelles, ils ont été choisis dans la même cohorte en 1980 et sont plus jeunes d'un an en 1981 . en 1981

L'alimentation a été distribuée à raison de $1,5 \%$ du poids vif en 1980 et ad libitum

Au cours de la deuxième année, tous les animaux ont été marqués individuellement (marque "Floy-tag ") afin de suivre leurs performances de reproduction au cours de la séquence ponte hivernale naturelle - ponte estivale sous photopériode contrôlée ponte hivernale sous photopériode naturelle.

\section{Structures d'élevage :}

Le travail a été réalisé à la pisciculture de MEILH LIVINOT (Finistère).

Les géniteurs sont maintenus dans des bassins circulaires de $4 \mathrm{~m}^{2}$ à évacuation centrale, équipés d'un couvercle opaque muni d'une lampe de 75 W alimentée en 24 volts. La durée journalière de l'éclairement est réglée par l'intermédiaire de programmateurs commerciaux type "flash-pratic".

Les bassins sont alimentés à partir d'un ruisseau dont la température de l'eau a varié journellement de 11 à $18^{\circ} \mathrm{C}$ pendant la période d'ovulation 1980 et n'a pas dépassé $15^{\circ} \mathrm{C}$ pendant celle de 1981 . Les deux années, la densité d'élevage a été de 50 géniteurs par bassin.

\section{Régimes photopériodiques :}

Les deux années, immédiatement après la ponte hivernale normale ( 20 janvier 1980 - 25 novembre 1980), les femelles et les mâles sont transférés sous une durée d'éclairement de 16 heures, puis soumis à une décroissance photopériodique de 16 à 8 heures de lumière en 6 mois, suivant au plus près la décroissance naturelle observée de juillet à décembre (Fig. 1a et b).

En 1980, l'expérience a été arrêtée dès après les pontes estivales, tandis qu'en 1981, elle a été poursuivie jusqu'à l'hiver, les animaux étant replacés dans des conditions photopériodiques naturelles aussitôt après l'ovulation estivale. Ils ont été transférés à la pisciculture fédérale d'ANTRAIN (IIle-et-Vilaine) en septembre 1981 où ils ont été conservés pour l'observation d'éventuelles pontes hivernales.

\section{Induction de ponte :}

L'intérêt des synchronisations de pontes par rapport aux ovulations naturelles a été analysé durant l'expérience réalisée en 1981. Dans ce but, à la date correspondant à environ $30 \%$ d'ovulations naturelles, les animaux restants ont été subdivisés en deux sous-groupes, l'un a ovulé naturellement, tandis que l'autre a été traité afin de tenter d'induire l'ovulation par traitement hormonal suivant le protocole décrit par JALABERT et al. (1978).

Dans ce but, les animaux ont reçu :

le 8 juillet 1981 une première injection de gonadotropine de Saumon partiellement purifiée selon BRETON et al. (1978), par voie intrapéritonéale à raison de $0,1 \mathrm{mg} / \mathrm{kg}$,

le 11 juillet une injection de $17 \alpha$-hydroxy-20 $\beta$-dihydroprogestérone administrée par la même voie à raison de $3 \mathrm{mg} / \mathrm{kg}$.

La détection des ovulations induites a eu lieu quatre jours plus tard le 15 juillet.

\section{PARAMÈTRES ÉTUdiés}

Les dates de ponte ont été détectées soit par contrôle bi-hebdomadaire du cheptel en 1980 à cause de la température élevée de l'eau, soit par un contrôle hebdomadaire en 1981.

Fertilité absolue après pesée du nombre total d'œufs. 


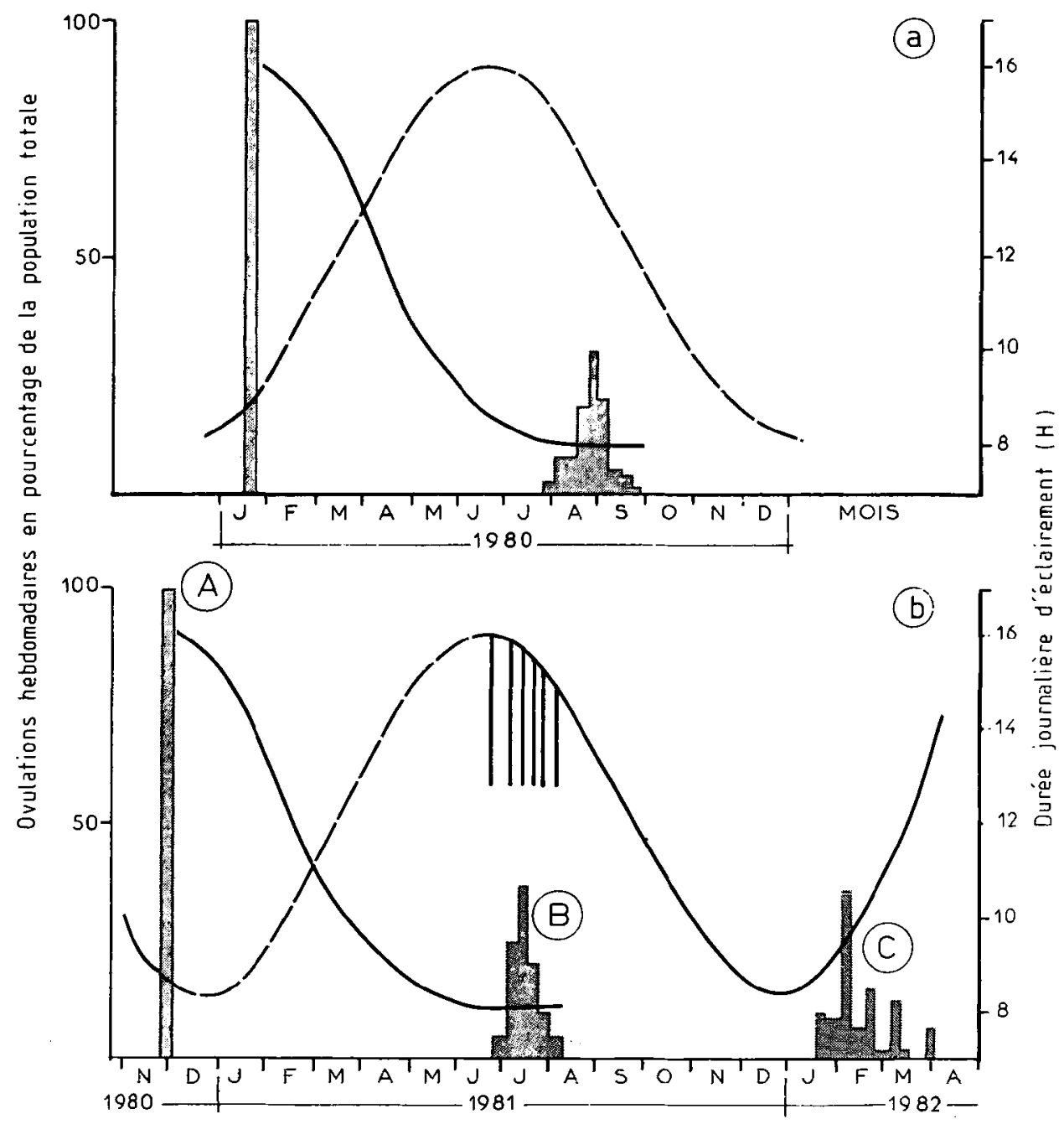

Figure 1 : Histogramme des fréquences d'ovulations en fonction de la date et durées journalières d'éclairement (courbe)
a - lors de l'expérimentation de 1980
b - lors de l'expérimentation de 1981.

Qualité des gamètes : 200 œufs prélevés individuellement sur chaque femelle ovulée ont été inséminés dans du dilueur " 532 " (BILLARD, 1977) par un mélange du sperme de 5 mâles à la dilution de 10-2. Ces cufs ont été transportés de la pisciculture au laboratoire dans de la glace pilée, puis après équilibrage naturel de la température à $12^{\circ} \mathrm{C}$ mis à incuber dans un circuit fermé thermorégulé à cette température. La qualité des ovules est estimée par le pourcentage d'œufs œillés à 220 degrés jours.

Ces deux derniers paramètres, fertilité et qualité des gamètes ont été comparés après ovulations naturelles et induites.

\section{Controle de la qualité des alevins}

10000 cufs œillés provenant de pontes non induites obtenues le 15 juillet 1981 ont été transférés à la pisciculture fédérale de CARDROC (IIle-et-Vilaine) où l'élevage des alevins a été suivi jusqu'au 30 mars 1982, et 20000 à la SODAB dans les Côtes-du-Nord. Le restant de ces mêmes pontes a été élevé à la pisciculture de MEILH LIVINOT. Les alevins ont été élevés dans ces trois piscicultures selon les pratiques en usage, sans directives de notre part. La croissance a été suivie par des pesées espacées d'environ 2 mois. 


\section{RÉSULTATS}

- Dates de pontes : (figures 1 a et b)

En 1980 comme en mai 1981, le traitement photopériodique a permis la ponte estivale de toutes les femelles. La première ovulation a lieu 159 jours après la mise sous photopériode contrôlée en 1980 et 193 jours en 1981 . Si l'on obtient une moyenne de 187 jours, $n=103$ (limites 159-211) en 1980 et de 208 jours, $n=49$ (limites 193-225) en 1981; les pics d'ovulation principaux se produisent à partir de 179 jours en 1980 et à partir de 205 jours en 1981, laissant apparaitre un décalage plus important en 1981 qu'en 1980, après la période d'ovulation hivernale. En 1981, recalée par rapport au solstice d'hiver auquel les animaux n'ont pas été soumis, la première ovulation se produit environ 167 jours après, valeur alors voisine des 159 jours après mise sous photopériodes décroissantes obtenue en 1980 lorsque les animaux y ont été placés après le solstice d'hiver.

Sur les 49 femelles ayant ovulé naturellement l'été 1981 après traitement photopériodique, 46 ont été remises sous photopériode naturelle. Elles ont toutes eu une nouvelle ponte hivernale en moyenne 214 jours après leur ponte estivale (limites : 184260).

Enfin, au cours de l'été 1981, les 24 femelles ayant été traitées pour l'induction hormonale de la ponte ont ovulé : 23,4 jours après la fin du traitement, la dernière une semaine plus tard. Dans tous les cas, la totalité des mâles a spermié et la spermiation a été contemporaine des ovulations.

Fortilité : (tableau 1, figure 2)

La fertilité absolue des femelles ovulant pour la $2^{\mathrm{e}}$ fois l'été 1980 ne diffère pas significativement de celle des femelles de même origine, ovulant pour la $2^{\theta}$ fois l'hiver $1981-1982$.

Sur la même cohorte d'animaux suivis de l'hiver $80-81$ à l'hiver $81-82$, la fertilité moyenne absolue augmente de façon très hautement significative. La ponte estivale est intermédiaire et significativement différente des deux pontes hivernales. Le traitement photopériodique n'apparaît donc pas altérer l'augmentation de la fertilité absolue, sans doute corrélée à la croissance globale des animaux.

Le traitement d'induction de ponte ne modifie pas significativement la quantité moyenne d'œufs pondus par animal.

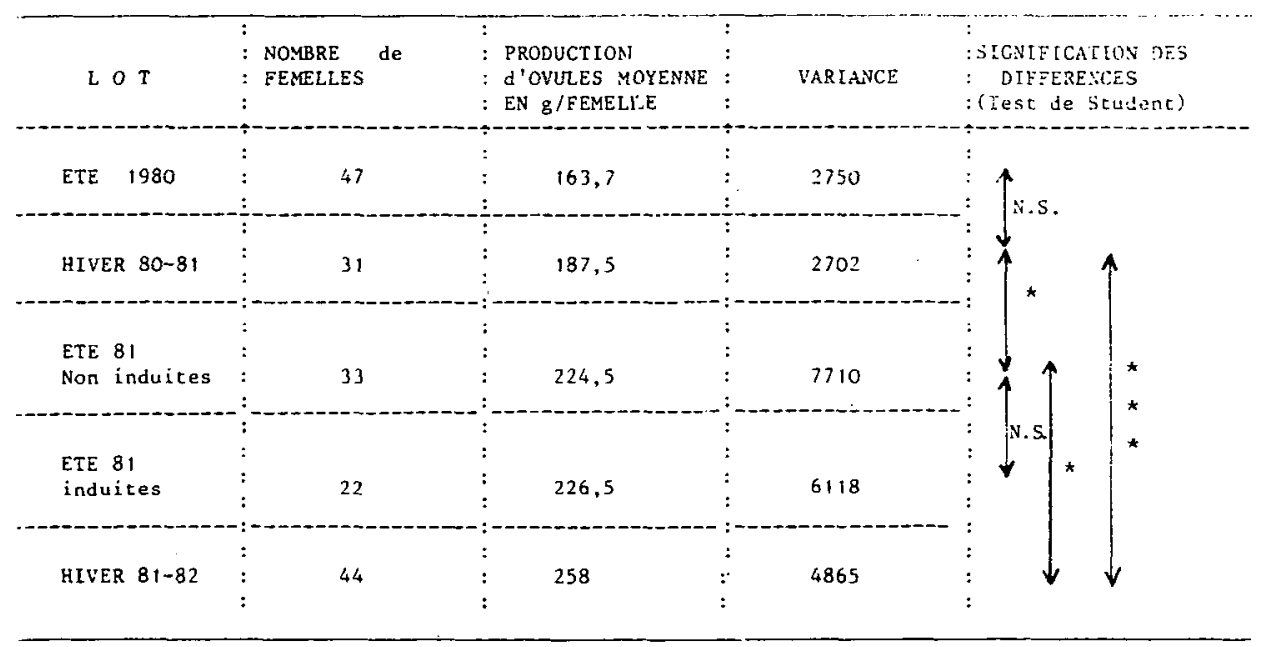

Tableau 1: Fertilités moyennes abs,olues des divers lots

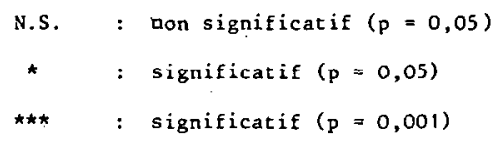



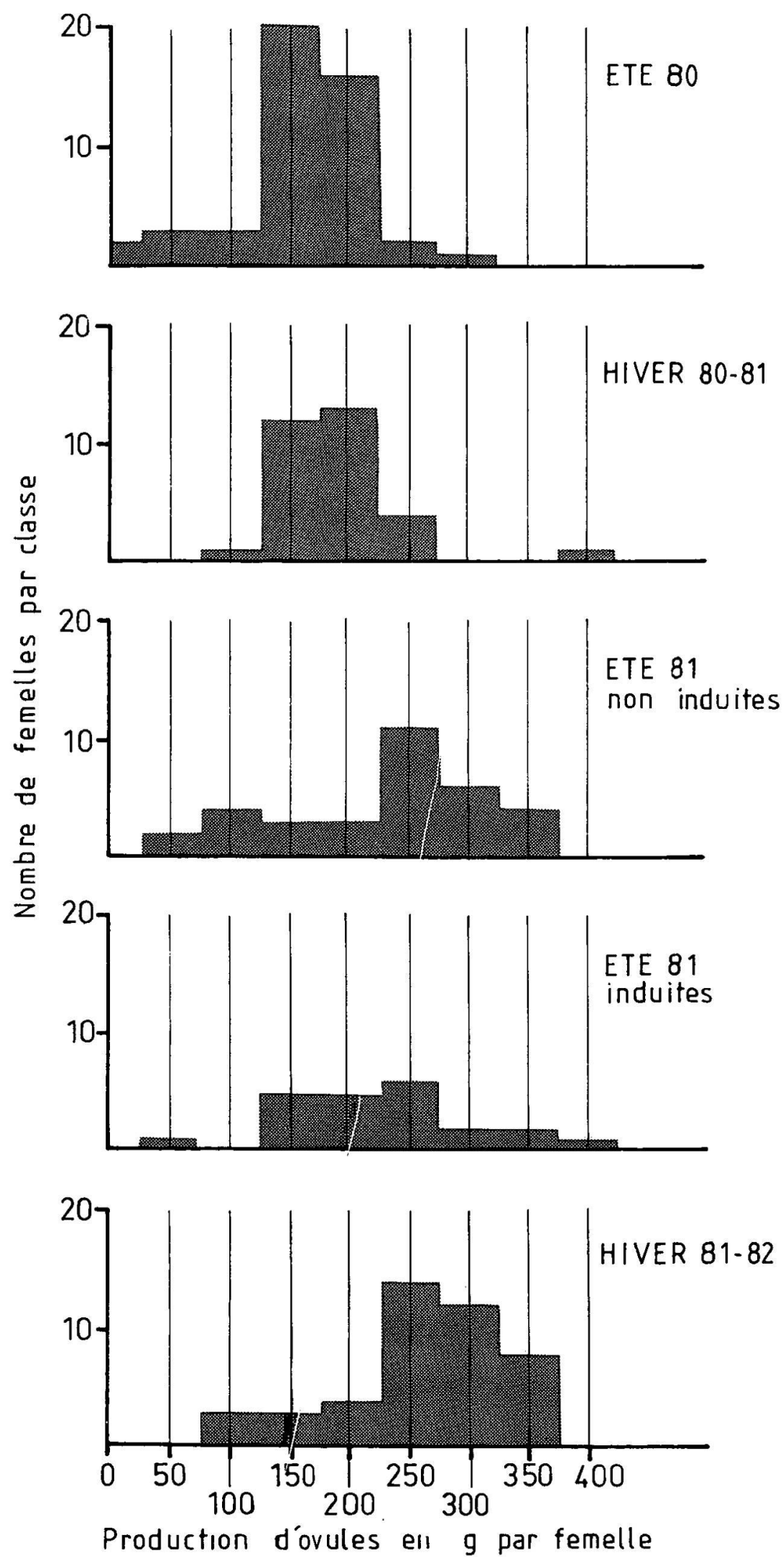

Figure 2 : Fertilités absolues en gramme d'ovules par femelle. 


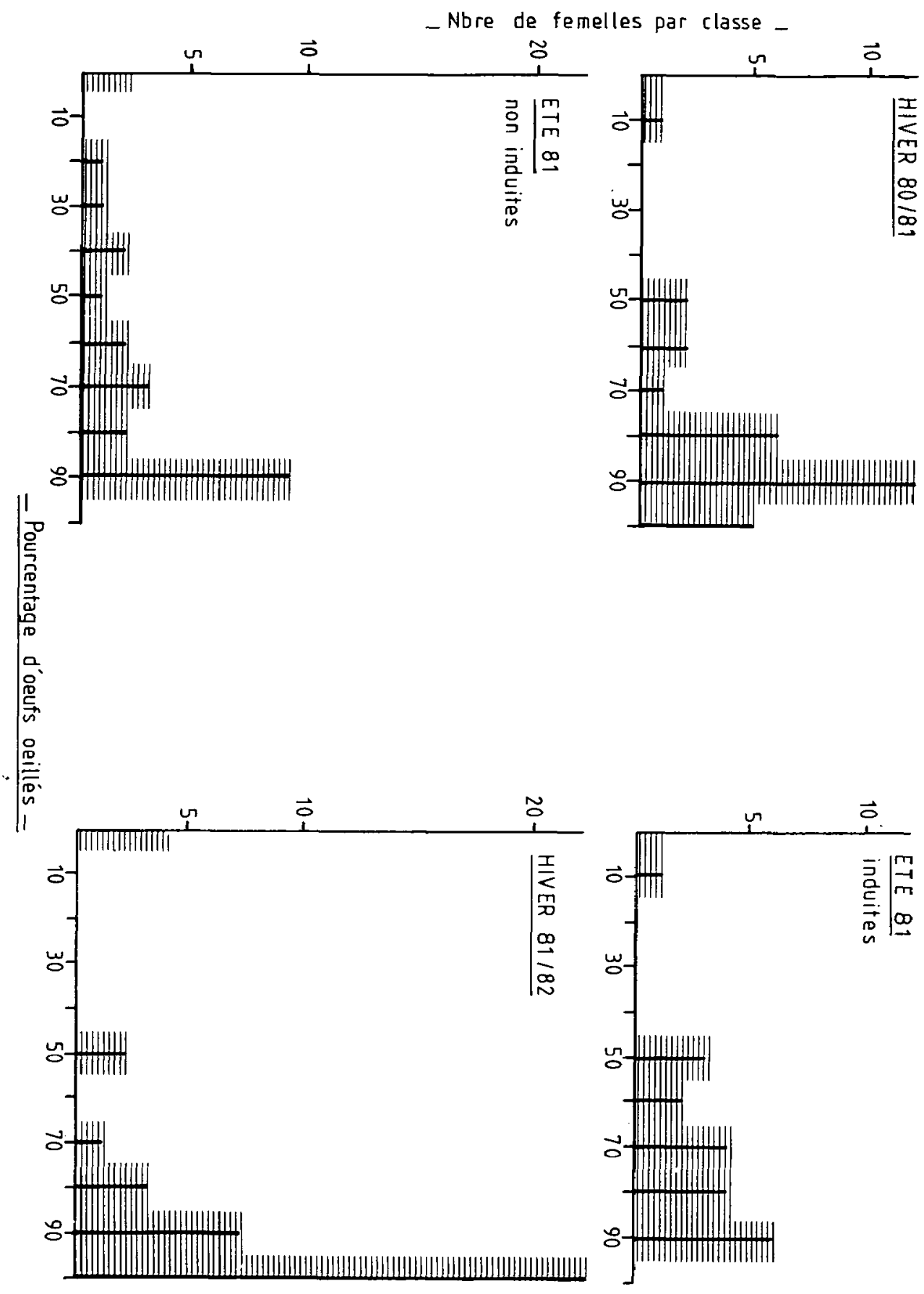

Figure 3 : Qualité des ovules produits jugée par le pourcentage d'coufs ceillés. 
Qualité des gamètes : (figure 3)

Les pontes de l'été 1980, durant lequel les températures diurnes ont atteint $18^{\circ} \mathrm{C}$ pendant la période d'ovulation, sont de qualité médiocre, avec un pourcentage moyen d'embryonnement à 220 degrés jours de $30 \%, 27 \%$ des animaux ont même présenté des pourcentages d'œufs œillés de $0 \%$, seuls $20 \%$ des sujets ont donné des pourcentages supérieurs à $50 \%$.

En 1981, les pontes estivales sont d'une qualité inférieure aux deux pontes hivernales qui les précèdent ou la suivent (tableau 2), au cours desquelles les pourcentages d'œuf œillés obtenus ne diffèrent pas significativement.

Les produits obtenus après hypophysation ne diffèrent pas significativement en moyenne de ceux obtenus après pontes naturelles. II faut cependant remarquer que le nombre d'animaux donnant des pourcentages de fécondation inférieurs à $50 \%$ est plus important après ponte naturelle qu'après ponte induite.

- Survie et croissance des alevins : (tableau 3, figure 4)

Dans les 3 piscicultures, la survie a été excellente et la croissance variable. Cette croissance, en fonction des degrés jours, est comparable à celle observée par FAURE (non daté) sur la même souche de Truite élevée dans une autre pisciculture bretonne. Le 30 mars 1981 , les truitelles avaient un poids moyen de $60 \mathrm{~g}$.

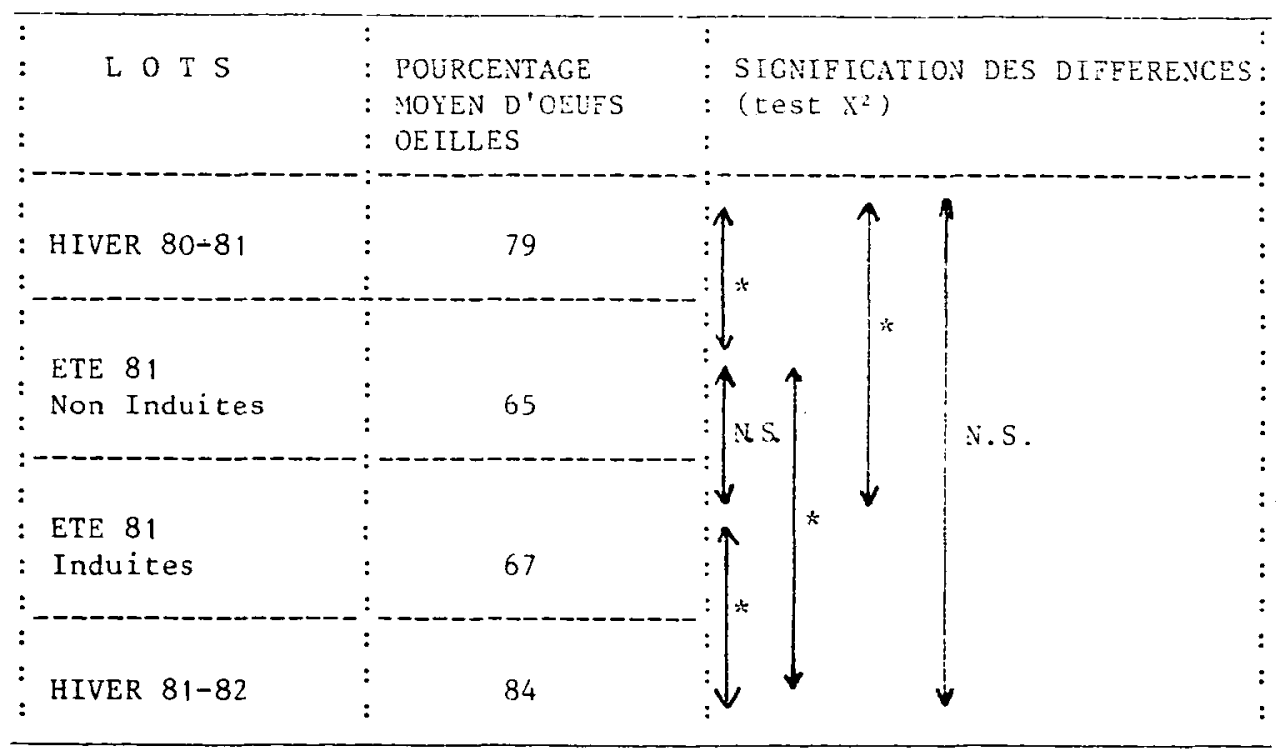

Tableau 2: Pourcentages moyens d'coufs coillés dans les pontes des différents lots.

(seuil de comparaison 75\%)

N.S. : non significatif $(p=0,05)$

* : significatif $(p=0,05)$ 


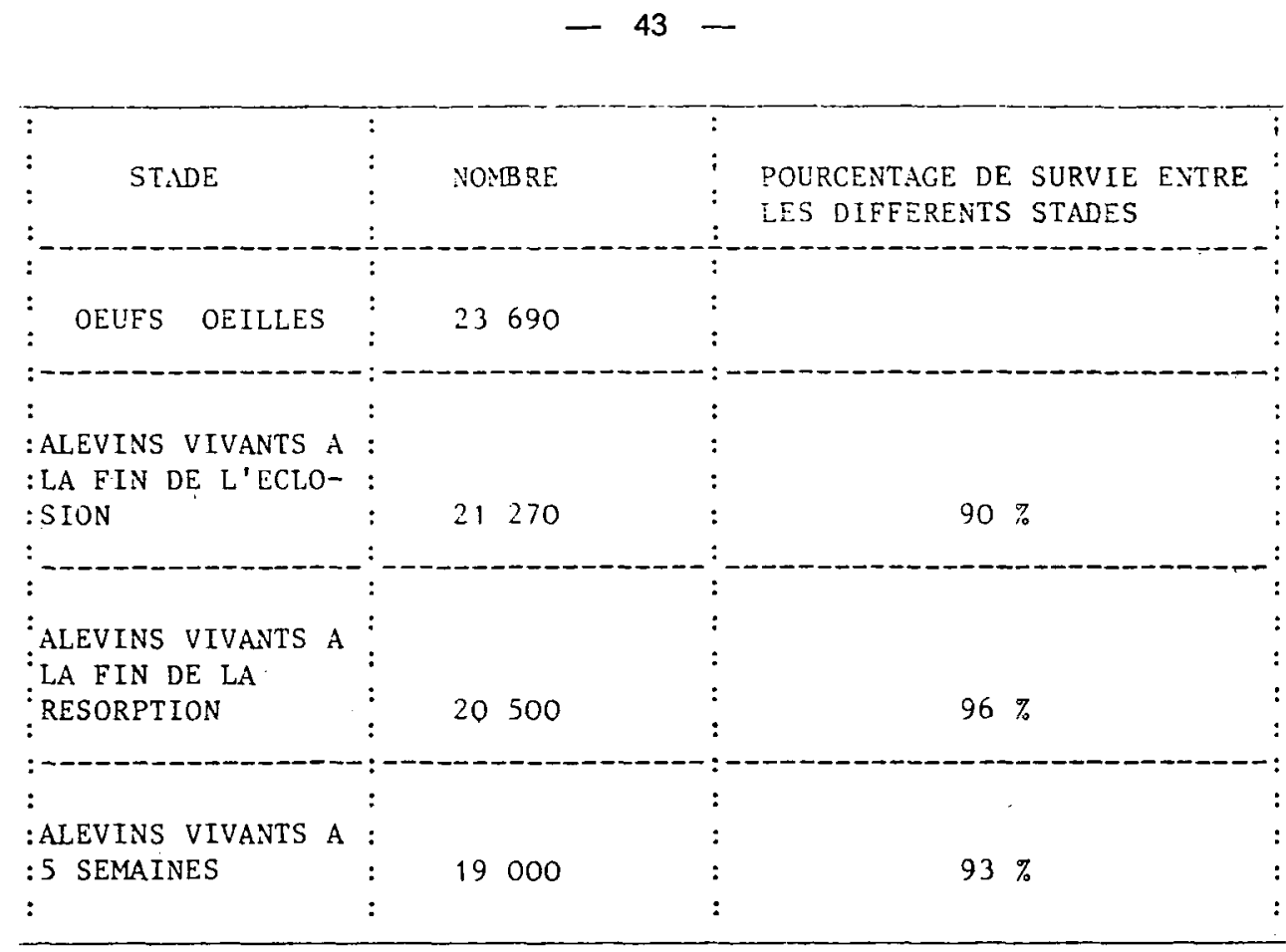

Tableau 3: Survie des alevins à la pisciculture de la SODAB (Données J.C. MAUVIOT).

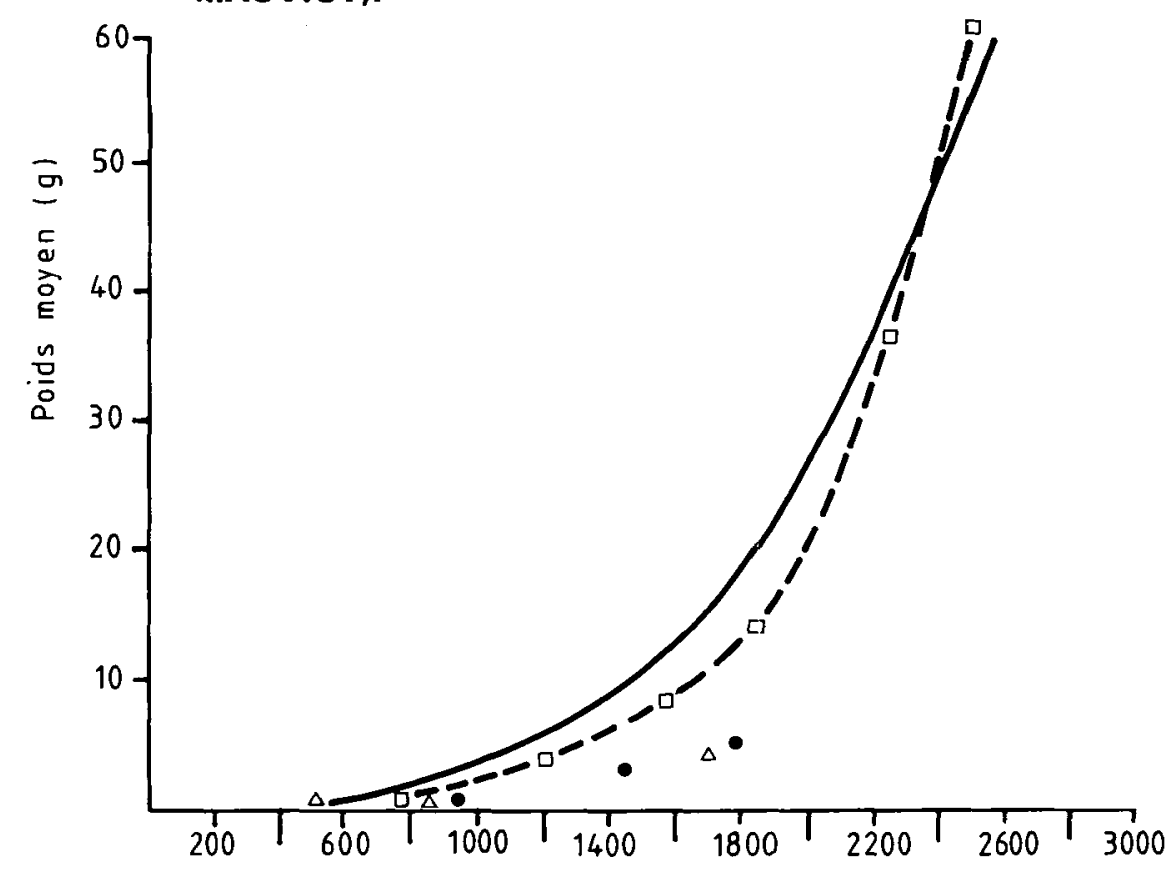

Somme des degrés jours depuis la fécondation

Figure 4 : Croissance en poids (g) des alevins en fonction de la somme des degrés jours depuis la fécondation.

Les symboles $\square \Delta \bullet$ caractérisent les différentes piscicultures. La courbe - est celle éteblie par FAURE (non daté) sur des Truites arc-en-çiel de la même souche.

L'équation de cette courbe est :

$P=11,38\left(\frac{0,26}{4500} \mathrm{DJ}\right)^{3} \times 1000$

Où P est le poids moyen en g et DJ la somme de degrés jours depuis la fécondation. 


\section{Discussion}

Ces expérimentations démontrent à l'échelle d'une unité de production piscicole la faisabilité d'un contrôle photopériodique de la reproduction déjà obtenu au laboratoire. Au cours des deux saisons consécutives, tous les animaux soumis au traitement photopériodique ont ovulé. Nos résultats antérieurs (BRETON et al., 1982) avaient démontré qu'après une ponte hivernale mi-décembre, il était possible d'obtenir un étalement des pontes de juillet à décembre en programmant, à partir du solstice d'hiver, la mise des animaux sous photopériode décroissante de 16 à $8 \mathrm{~h}$. de lumière en six mois : la ponte intervenait généralement six mois et demi après le début de la phase de contrôle. Nos résultats obtenus au cours de l'expérience réalisée en 1980 confirment ces résultats antérieurs. Par contre, notre objectif 1981, obtention de pontes début juin pour éviter les hautes températures estivales, $n$ 'a pas pu être atteint avec la souche de Truite arc-en-ciel utilisée. Bien que le traitement photopériodique ait débuté le 25 novembre, la première ponte estivale n'a eu lieu que le $\mathbf{2 6}$ juin, soit après sept mois. Ces résultats pourraient signifier que les Truites ne peuvent réinitier un nouveau cycle de gamétogénèse tant qu'elles n'ont pas été soumises à l'inversion photopéridique du solstice, signal mémorisable nécessaire à une reprise qui peut alors se faire indépendemment de la forme de la photopériode (BRETON et al., 1982). Certains auteurs (SKARPHEDISSON et al., 1982) ont suggéré que c'est la longueur du jour au printemps qui initierait la gamétogénèse. L'ensemble de nos présents résultats et de ceux obtenus antérieurement ne vont pas à l'encontre de cette hypothèse, que nous pensons plus probable que celle impliquant l'intervention nécessaire des jours longs pour qu'il y ait reprise de la gamétogénèse (BROMAGE et al., 1982). Dans ce domaine, la découverte de la longueur d'une phase photosensible serait sans doute primordiale pour les possibilités pratiques de contrôle du cycle qu'elle ouvrirait.

Bien qu'au cours des deux années d'expérience tous les animaux aient été sélectionnés pour avoir ovulé dans la même semaine, les ovulations estivales s'étendent sur 62 jours, l'été 80 et 32 jours l'été 81 . On note donc une dispersion qui pourrait correspondre à des différences entre la vitesse de déroulement de la vitellogénèse ou des processus de maturation des femelles. En admettant une durée de vitellogénèse constante, ces dernières valeurs indiqueraient que la durée des phases finales de maturation des gamètes serait plus courte à basse température (62 jours en 80 température maximale diurne $18^{\circ} \mathrm{C}-32$ jours en 81 , température maximale diurne $\left.15^{\circ} \mathrm{C}\right)$.

Outre cet effet possible de la température sur la dynamique de la maturation, ce facteur doit affecter la survie des œufs post ovulation, phénomène déjà observé chez l'Omble de fontaine (HOKANSON et al., 1973) et la Truite arc-en-ciel (BILLARD et BRETON, 1977). En effet, lorsque les animaux ont été soumis à des maxima diurnes de $18^{\circ} \mathrm{C}$, le pourcentage moyen de fécondation a été de $30 \%$ alors que, si ce maximum ne dépasse pas $15^{\circ} \mathrm{C}$, les pourcentages d'embryonnements moyens à 220 degrés jours sont voisins de $70 \%$ mais inférieurs aux valeurs hivernales. HOKANSON avait donné $16^{\circ} \mathrm{C}$ comme température limite supérieure de survie des œufs chez l'Omble, cette température pourrait être identique chez la Truite dans la mesure où à $15^{\circ} \mathrm{C}$ on observe déjà une chute des pourcentages de fécondation, observation corroburée par le fait qu'à $15^{\circ} \mathrm{C}$ après induction de ponte, lorsque l'on peut prévoir la date d'ovulation et éviter le vieillissement intra abdominal des œufs, le nombre d'animaux présentant des pourcentages de fécondation inférieurs à $50 \%$ est plus faible qu'après une ponte naturelle détectée deux fois par semaine. Ainsi, si l'on utilise des eaux présentant de fortes amplitudes des variations journalières de température, l'induction de la ponte devient un outil puissant pour l'obtention de produits sexuels conservant une bonne fécondabilité.

\section{CONCLUSION}

En l'état actuel de nos connaissances, il ne paraît pas possible, à partir de souches de Truites arc-en-ciel à ponte hivernale, d'obtenir de nouvelles ovulations avant le début du mois de juillet suivant par contrôle photopériodique.

Par contre, à partir de cette date, il est possible de programmer les pontes en utilisant une décroissance photopériodique de 16 à $8 \mathrm{~h}$ de lumière en six mois, les dates d'ovulations se produisent généralement dans les deux semaines suivant la fin du traitement photopériodique. Ces traitements ne paraissent applicables qu'à des animaux élevés dans des eaux présentant des maximums estivaux inférieurs à $15^{\circ} \mathrm{C}$. L'utilisation 
de l'induction hormonale de la ponte peut partiellement solutionner ce problème et également permettre d'obtenir une synchronisation des pontes, élément de gestion piscicole important. Dans ces conditions, le contrôle photopériodique de l'époque de la ponte paraît d'ores et déjà pouvoir être appliqué dans la pratique piscicole.

\section{REMERCIEMENTS}

Le présent travail a fait l'objet d'une aide à l'innovation de la part del'A.N.V.A.R. BRETAGNE.

Madame LEMENN nous a apporté son aide tout au long de ces expérimentations.

J.C. MAUVIOT (SODAB) et V. FOLMER (Fédération des A.A.P.P. d'Ille-et-Vilaine) ont eu la responsabilité du testage des alevins dans leur pisciculture respective.

C. MICHAUD (Fédération des A.A.P.P. d'Ille-et-Vilaine) a assuré l'entretien des géniteurs dans la pisciculture d'ANTRAIN.

\section{BIBLIOGRAPHIE}

BILLARD R.; 1977. Utilisation d'un système tris-glycocolle poụr tamponner le dilueur d'insémination de Truite. Bull. Fr. Pisc. 264, 102-112.

BILLARD R., BRETON B., 1977. Sensibilité à la température des différentes étapes de la reproduction chez la Truite arc-en-ciel. Cahiers Lab. Hydrobiol. Montereau, 5, 523.

BRETON B., BILLARD R., 1977. Effects of photoperiod and temperature on plasma gonadotropin and spermatogenesis in the rainbow trout Salmo gairdneri R. Ann. Biol. anim. Biochem. Biophys. 17, 331-340.

BRETON B., PRUNET P., REINAUD P., 1978. Sexual differences in salmon gonadotropin. Ann. Biol. anim. Biochem. Biophys. 18, 754-765.

BRETON B., ZOHAR Y., BILLARD R., 1982. Photoperiod and gonadotropin control of the reproductive cycle in the female rainbow trout. Proceeding of the 9 th Intern. Symp. on Comparative Endocrinology. B. LOFT ed. Hong-Kong University Press.

BROMAGE N.R., WHITEHEAD E., ELLIOT J. AK., BRETON B., MATTY A.J., 1982. Investigations into the importance of daylength on the photoperiodic control of reproduction in the female rainbow trout. In Reproductive physiology of Fish. 233-236 C.J.J. RICHTER, H.J. Th. GOOS eds, Pudoc, Wageningen (The Netherlands).

FAURE A. (non daté). Bases biotechniques et économiques de mise en place d'une gestion prévisionnelle en aquaculture - exemple de la salmoniculture C.T.G.R.E.F./ I.N.A. P.G. Cycle supérieur de formation sur le développement des productions animales en milieux aquatiques, $12 \mathrm{p}$.

HOKANSON K.E.F., Mc CORMICK J.H., JONES B.R., TOCKER J.H., 1973. Thermal requirements for maturation, spawning and embryo survival of the brook trout Salvelinus fontinalis. J. Fish. Res. Board. Can. 30, 975-984.

HOOVER E.E., HUBBARD H.E., 1937. Modification of the sexual cycle in trout by control of light. Copeia, 4, 206-211.

HTUN HAN M., 1977. The effects of photoperiod on reproduction in fishes - an annotated bibliography Library information leaflet $n^{\circ} 6$. Lowestoft.

JALABERT B., GOETZ F.W., BRETON B., FOSTIER A., DONALDSON E.M., 1978. Precocious induction of oocyte maturation and ovulation in coho salmon Oncorhynchus kisutch. J. Fish. Res. Board. Can. 35, 1423-1429.

NOMURA M., 1962. Studies on reproduction of rainbow trout Salmo gairdneri with special reference to egg taking. III. Acceleration of spawning by control of light. Bull. Jap. Soc. Scient. Fish. 28, 1070-1076.

SKARPHEDISSON O., SCOTT A.P., BYE V.J., 1982. Long photoperiods stimulate gonad development in rainbow trout. In Reproductive Physiology of Fish. p. 243. C.J.J. RICHTER, H.J. Th. GOOS eds, Pudoc, Wageningen (The Netherlands).

SUNDARARAJ B.I., 1981. Reproductive physiology of teleost fishes. A review of present knowledge and need for future research. FAO. Aquaculture development and coordination program. ADCP/REP/81/16. 1-82.

WHITEHEAD C., BROMAGE N.R., FORSTER J.R.M., MATTY A.J., 1978. The effects of alteration in photoperiod on ovarian development and spawning time in the rainbow trout Salmo gairdneri. Ann. Biol. anim. Biochem. Biophys. 18, 1035-1043. 